\title{
Changes of primary headache related white matter lesions in pediatric patients
}

\author{
Erhan Bayram ${ }^{1}$, Uluç Yiş ${ }^{1}$, Cem Paketçi ${ }^{1}$, Derya Okur ${ }^{1}$, İpek Polat ${ }^{1}$, Handan Çakmakc1 ${ }^{2}$, \\ Semra Hiz ${ }^{1}$, Banu Anlar ${ }^{3}$ \\ Division of ${ }^{1}$ Pediatric Neurology, Department of Pediatrics and ${ }^{2}$ Department of Radiology, Dokuz Eylül University Faculty \\ of Medicine, Izmir; ${ }^{3}$ Division of Pediatric Neurology, Department of Pediatrics, Hacettepe University Faculty of Medicine, \\ Ankara, Turkey. E-mail: dr.erhanbayram@yahoo.com \\ Received: 29th December 2017, Revised: 4th March 2018, Accepted: 18th March 2018
}

SUMMARY: Bayram E, Yiş U, Paketçi C, Okur D, Polat İ, Çakmakcı H, Hız $S$, Anlar B. Changes of primary headache related white matter lesions in pediatric patients. Turk J Pediatr 2018; 60: 380-384.

We aimed to describe the long-term prognosis of white matter lesions detected on magnetic resonance imaging in children with primary headache. Children who were admitted with the complaint of headache and had nonspecific white matter lesions on magnetic resonance imaging were included in the study. The clinical findings of the patients were reinvestigated using the same magnetic resonance imaging scanner and acquisition protocol after at least a two year period. Magnetic resonance imaging results of the patients were documented in detail. Findings of the baseline and follow-up studies were compared with each other by the same radiologist. Among the 11 patients, 8 ( $72.7 \%$ ) were male and $3(27.3 \%)$ were female. Mean age of patients at the time of second imaging was $12.9 \pm 2.3$ years. Eight $(72.7 \%)$ had migraine without aura, $1(9.1 \%)$ had tension-type headache and $2(18.2 \%)$ had migraine with aura. The mean clinical follow-up period of the patients was $4.31 \pm 1.31$ years. All patients had low headache frequency on the last control visit when compared to the first clinical findings. The follow-up magnetic resonance imaging studies showed two newly developed white matter lesions in two patients who had migraine without aura and the white matter lesions disappeared in the patient who had tension-type headache, compared to the baseline neuroimaging. Findings of the baseline and long-term follow-up magnetic resonance imaging studies of the patients with primary headache showed no significant changes in terms of the location, size and laterality. Repeated neuro-imaging studies are not warranted in the absence of the progression in clinical findings.

Key words: white matter lesions, headache, children, magnetic resonance imaging, longterm evaluation.

Primary headaches are common complaints in pediatric population. The reported rate of migraine prevalence before the age of 15 is $11 \% .^{1}$ Brain magnetic resonance imaging (MRI) studies of the patients with chronic primary headache, frequently showed white matter lesions (WMLs). 2,3 The relationship between primary headache and WMLs is not completely understood. Previous studies showed an increase risk for subclinical brain infarcts and WMLs in patients especially with migraine with aura. 4,5 The rate of incidental non-specific WMLs on MRI was $4.4 \%$ in children with headache.

In this study we aimed to describe the longterm prognosis of WMLs according to the headache duration and frequency of the primary headache.

\section{Material and Methods}

This study was conducted in the Pediatric Neurology Department of Dokuz Eylul University, Izmir, Turkey. Patients with chronic primary headache who were diagnosed according to the International Classification of Headache Disorders and nonspecific WMLs on 
brain MRI were called for a control visit and included the study. Current status of headache, and neurologic findings were determined. The patients were reinvestigated after at least two years period by using the same MRI scanner and acquisition protocol. ${ }^{6}$ Patients with chronic primary headache and nonspecific WMLs on brain MRI with a follow up time less than 2 years, were excluded from the study.

All MRI examinations were performed on a 1.5 Tesla magnet (Gyroscan Intero, Philips, Best, the Netherlands). T1-weighted spin-echo images were obtained from coronal, axial, and sagittal plane using a slice thickness of 4-7 $\mathrm{mm}$ and turbo spin-echo T2-weighted images were obtained from coronal and axial plane with a slice thickness of $5-7 \mathrm{~mm}$.

WMLs detected on MRI were classified according to their location such as supratentorial, infratentorial, periventricular white matter, and subcortical white matter. Laterality (unilateral or bilateral), number and sizes of the lesions, number of the newly developed WMLs and disappeared WMLs were also recorded. All of the MRI findings of the patients, performed at least two years apart, were reevaluated by the same pediatric neuroradiologist.

Informed consent forms were taken from all participants and all procedures were approved by the local ethical committee. (15/06/2017, 2017/16-20).

\section{Results}

Among the 11 patients, $8(72.7 \%)$ were male and $3(27.3 \%)$ were female. Mean age of the patients at the time of first imaging was $8.7 \pm 1.6$ years and $12.9 \pm 2.3$ years at the time of second imaging. According to the International Classification of Headache Disorder, $8(72.7 \%)$ had migraine without aura, $1(9.1 \%)$ had tension-type headache and $2(18.2 \%)$ had migraine with aura. The mean clinical follow-up period of the patients was $4.31 \pm 1.31$ years. All patients had normal psychomotor development, and there was no history of seizures or head trauma. The physical
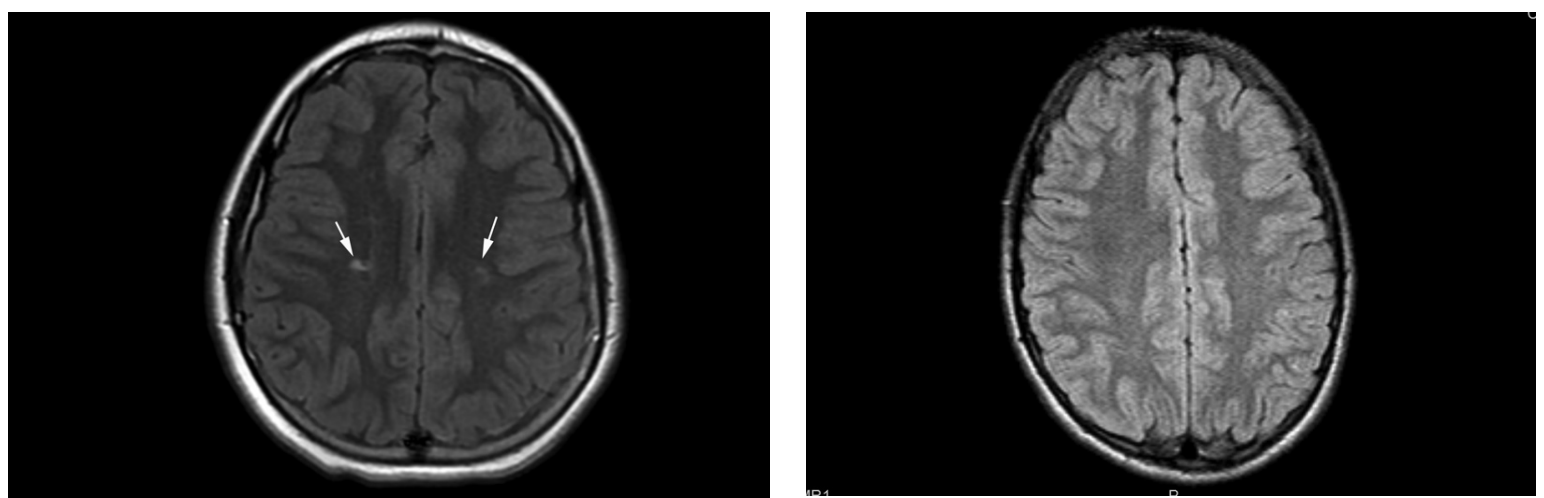

Fig. 1. Initial (a) and follow up (b) brain MRI FLAIR images show right cerebral white matter lesion (arrow on a) disappeared on follow up MR exam.
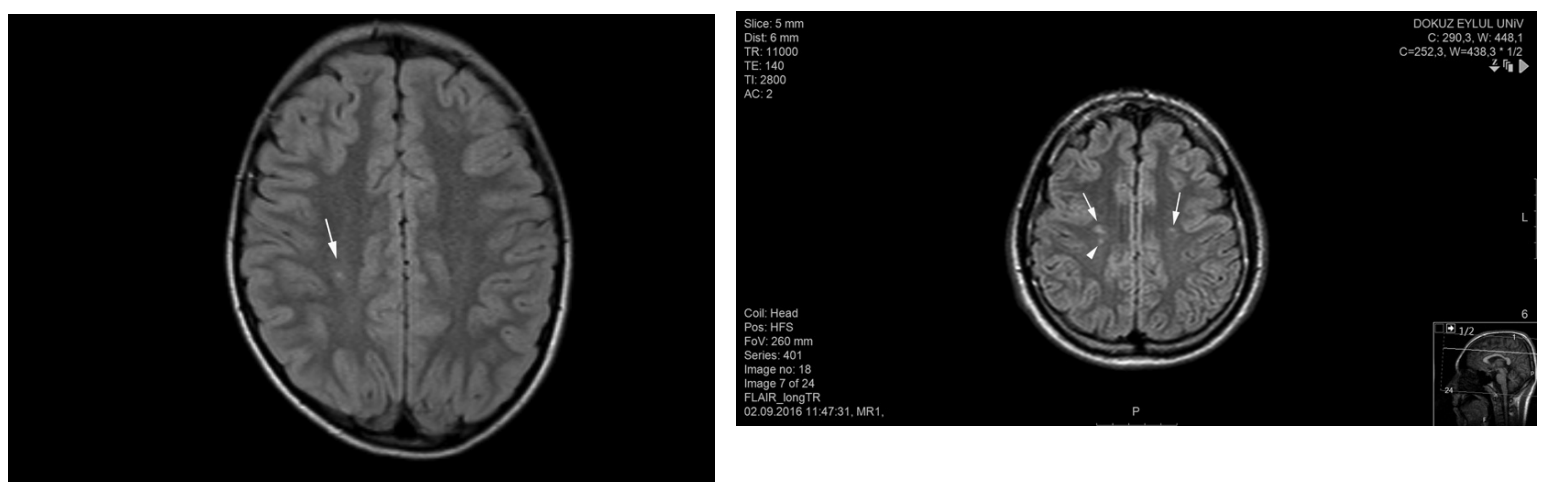

Fig. 2. Initial (a) and follow up (b) brain MRI FLAIR images show bilateral white matter lesions (arrows on a and b). There is a new white matter lesion (arrowhead on b) seen on follow up MR exam. 


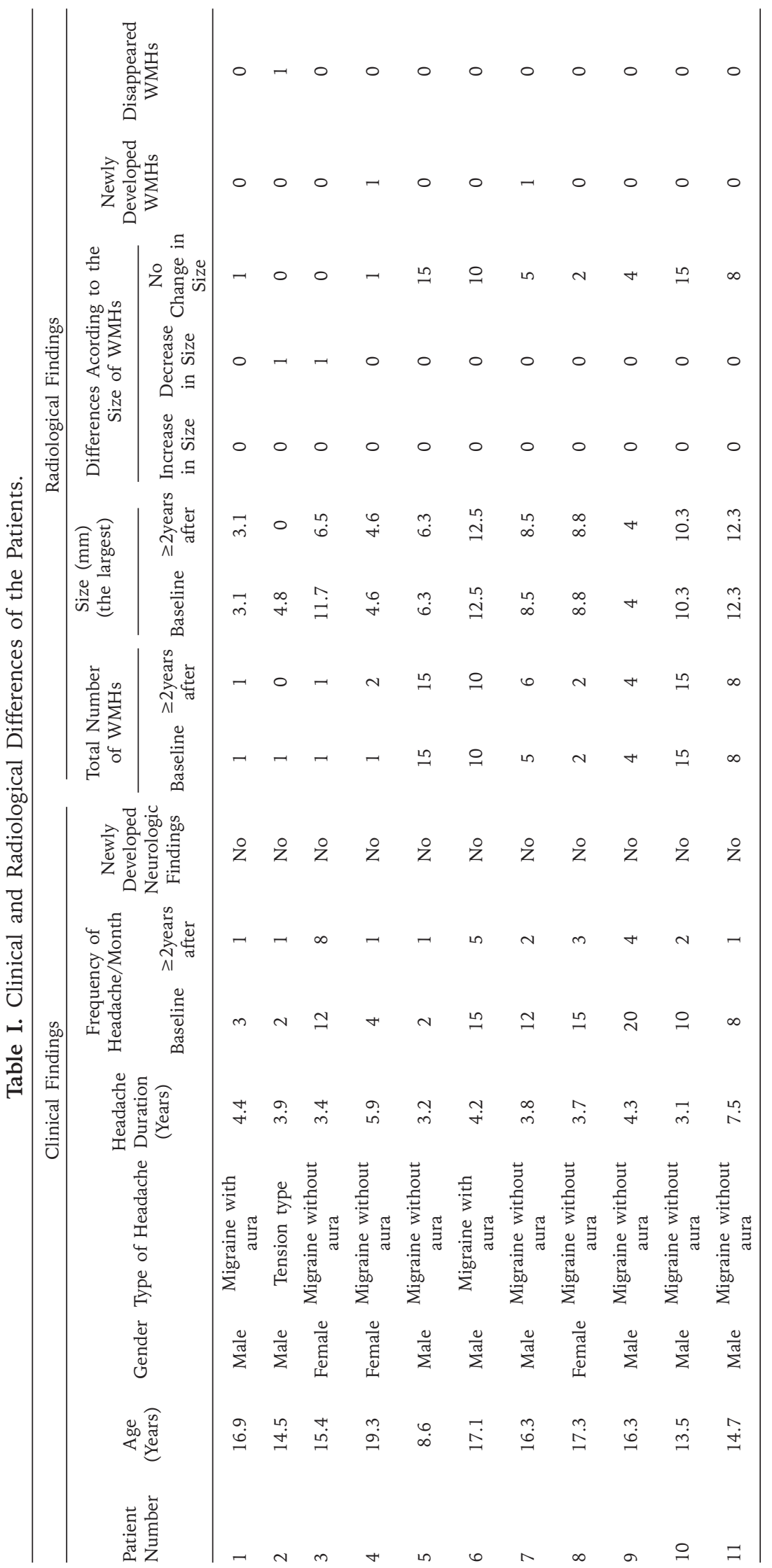


Table II. Differences According to the WMLs Distribution on MRI Findings.

\begin{tabular}{|c|c|c|c|c|c|c|c|}
\hline \multirow{2}{*}{$\begin{array}{l}\text { Patient } \\
\text { number }\end{array}$} & \multicolumn{2}{|c|}{ Supratentorial } & \multicolumn{2}{|c|}{ Infratentorial } & \multicolumn{2}{|c|}{ Laterality } & \multirow{2}{*}{$\begin{array}{c}\text { Lobar distribution } \\
\text { differences }\end{array}$} \\
\hline & Baseline & $\begin{array}{l}\geq 2 \text { years } \\
\text { after }\end{array}$ & Baseline & $\begin{array}{c}\geq 2 \text { years } \\
\text { after }\end{array}$ & Baseline & $\geq 2$ years after & \\
\hline 1 & + & + & - & - & Unilateral & Unilateral & - \\
\hline 2 & + & - & - & - & Unilateral & - & - \\
\hline 3 & - & - & + & + & Unilateral & Unilateral & - \\
\hline 4 & + & + & - & - & Unilateral & Bilateral & + \\
\hline 5 & + & + & - & - & Bilateral & Bilateral & - \\
\hline 6 & + & + & - & - & Bilateral & Bilateral & - \\
\hline 7 & + & + & - & - & Bilateral & Bilateral & - \\
\hline 8 & + & + & - & - & Unilateral & Unilateral & - \\
\hline 9 & + & + & - & - & Bilateral & Bilateral & - \\
\hline 10 & + & + & - & - & Bilateral & Bilateral & - \\
\hline 11 & + & + & - & - & Bilateral & Bilateral & - \\
\hline
\end{tabular}

MRI: Magnetic resonance imaging; WMLs: White matter lesions

examinations of all patients were normal. There was no patient under the therapy of migraine prophylaxis.

The mean number of the lesions was 5.73 and 5.82 on the first and second MRI scans respectively $(p=0.59)$. The mean size of the lesions was $7.9 \pm 3.5 \mathrm{~mm}$ (minimum, 3.1 $\mathrm{mm}$; maximum, $12.5 \mathrm{~mm}$ ) and $6.99 \pm 3.92$ $\mathrm{mm}$ (minimum, $3.1 \mathrm{~mm}$; maximum, 12.5 $\mathrm{mm}$ ) on the first and second MRI scans respectively $(p=0.17)$. The follow-up MRI studies showed two newly developed WMHs in two patients who had migraine without aura and in the patient with tension-type headache the WML disappeared, compared to the baseline neuroimaging (Table I), (Fig. 1-2).

A decrease in the size of WMLs was detected in only two patients (one disappeared and one decrease) and in nine patients the size of WMLs did not change, no patients showed increased in size, compared to the baseline neuroimaging. All patients had low headache frequency on the last control visit compared to the first clinical findings (Table I).

The location of WMLs was infratentorial only in one patient and was supratentorial in nine patients; in one patient who had supratentorial WML on first MRI scan, the WML had disappeared on the last MRI scan.
In seven patients WMLs were bilateral and in three patients WMLs were unilateral. In only one patient who had unilateral WMLs on the first neuroimaging, studies developed new WMLs on the last neuroimaging studies. There were no significant differences in terms of location and laterality of WMLs between the first and the last neuroimaging findings (Table II).

\section{Discussion}

Headache is a common symptom in children with various clinical conditions. The prevalence of chronic primary headache in childhood period is $25 \%$ and migraine and tension-type headaches are the common types. ${ }^{7}$ Several studies have reported an association between headache and WMLs on brain MRI in pediatric and adult patients. ${ }^{3,6,8}$ The studies of Bashir et al. ${ }^{9}$ showed an association between WMLs and migraine with aura but not for migraine without aura. In contrast with the previous studies Gaist et al. ${ }^{10}$ concluded that migraine with aura did not increase the risk of silent infarcts and also these WMLs did not significantly differ in migraine group compared with non-migraine control group. It was reported that WMLs are found in $10 \%$ of children with migraine and in our previous study the rate of WMLs in pediatric patients with primary headache was 
$4.4 \% .^{3,6}$ In the present study, we reinvestigated the previously studied pediatric headache group with brain WMLs and we aimed to describe the long term prognosis of white matter lesions, at least two years after the initial neuroimaging.

The headache duration and the migraine attack frequency have a role in the WMLs evolution. Cerebral hemodynamic changes due to the repeated headache attacks have been considered in the formation of white matter changes. ${ }^{11,12}$ Szilvia et al. ${ }^{13}$ investigated the changes of migraine-related white matter hyperintensities in the same adult group after a three year interval and they concluded that small WMLs in patients with a low migraine attack frequency had a higher chance to disappear than large WMLs or WMLs in patients with a high attack frequency. They detected no meaningful changes in the hemispheric and lobar distribution of hyperintensities. ${ }^{13}$ Similar with these findings, in our study group, there was no significant differences between the baseline and the followup studies for the intracerebral distribution of WMLs. In our study group, only two patients (both patients were migraine without aura) had progressive and one patient (tension type of headache) had regressive WMLs in number. No patient had progressive WML in size. Headache related WMLs were more static in our study group. This may be due to the decreased headache frequency of all patients during at least a two year period.

The limitations of this study include the small sample size and having data from a single center.

In conclusion, non-specific incidental white matter changes may be seen in children with chronic primary headache. Findings of the baseline and long-term follow-up MRI studies of the patients with headache and cerebral WMHs showed no significant changes according to the hemispheric, lobar, supratentorial and infratentorial distribution. Repeated neuroimaging studies are not warranted in the absence of the neurological deterioration.

\section{REFERENCES}

1. Mortimer MJ, Kay J, Jaron A. Epidemiology of headache and childhood migraine in an urban general practice using Ad Hoc, Vahlquist and IHS criteria. Dev Med Child Neurol 1992; 34: 1095-1101.

2. Cooney BS, Grossman RI, Farber RE, Goin JE, Galetta SL. Frequency of magnetic resonance imaging abnormalities in patients with migraine. Headache 1996; 36: 616-621.

3. Eidlitz-Markus T, Zeharia A, Haimi-Cohen Y, Konen O. MRI White matter lesions in pediatric migraine. Cephalalgia 2013; 33: 906-913.

4. Kurth T, Mohamed S, Maillard P, et al. Headache, migraine, and structural brain lesions and function: Population based Epidemiology of Vascular AgeingMRI study. BMJ 2011; 342: c7357.

5. Kruit MC, Launer LJ, Ferrari MD, van Buchem MA Infarcts in the posterior circulation territory in migraine. The population-based MRI CAMERA study. Brain J Neurol 2005; 128(Pt 9): 2068-2077.

6. Bayram E, Topcu Y, Karaoglu P, Yis U, Cakmakci Guleryuz H, Kurul SH. Incidental white matter lesions in children presenting with headache. Headache 2013; 53: $970-976$.

7. Brna PM, Dooley JM. Headaches in the pediatric population. Semin Pediatr Neurol 2006; 13: 222-230.

8. Swartz RH, Kern RZ. Migraine is associated with magnetic resonance imaging white matter abnormalities: A meta-analysis. Arch Neurol 2004; 61: 1366-1368.

9. Bashir A, Lipton RB, Ashina S, Ashina M. Migraine and structural changes in the brain: A systematic review and meta-analysis. Neurology 2013; 81: 1260-1268.

10. Gaist D, Garde E, Blaabjerg M, et al. Migraine with aura and risk of silent brain infarcts and white matter hyperintensities: An MRI study. Brain 2016; 139(Pt 7): 2015-2023.

11. Trauninger A, Leel-Ossy E, Kamson DO, et al. Risk factors of migraine-related brain white matter hyperintensities: An investigation of 186 patients. J Headache Pain 2011; 12: 97-103.

12. Kruit MC, Launer LJ, Ferrari MD, van Buchem MA Brain stem and cerebellar hyperintense lesions in migraine. Stroke 2006; 37: 1109-1112.

13. Erdelyi-Botor S, Aradi M, Kamson DO, et al. Changes of migraine-related white matter hyperintensities after 3 years: A longitudinal MRI study. Headache 2015; 55: $55-70$. 